\title{
Separation of Noise from Distortion for High-Speed Optical Fiber System Link Budgeting
}

\author{
R. Hui, Senior Member, IEEE, M. Vaziri, J. Zhou, and M. O'Sullivan
}

\begin{abstract}
In optical transmission systems and networks with in-line optical amplifiers, system performance degradations caused by random noise and optical path distortions are usually thought to be inseparable, which makes link performance evaluation complicated. We propose to use an optical system eye-mask parameterization technique to separate noise from distortion in the link budgeting. The basic idea of this approach is to find the worst-case path distortion factor which is independent of the noise characteristics and system implementations. Both theoretical development and experimental verification are discussed.
\end{abstract}

Index Terms-Distortion, optical fiber communications, optical noise, optical system link budgeting.

\section{INTRODUCTION}

T O EVALUATE the performance of an optical transmission system, a quality factor $Q$, which is directly related system bit-error rate (BER), is often used [1]. In system $Q$ calculations, distortion, and noise are interrelated [2]. This complicates the practice of system link budgeting by obscuring the origins of system performance degradation. This problem is particularly acute when a system is comprised of equipment from different manufacturers. In such cases, interface specifications and trouble isolation are especially important. By way of example, a 1-dB eye distortion in an optical system gives rise to a power penalty of between 1 and $2 \mathrm{~dB}$ depending on the ratio of signal-dependent noise and signal-independent noise.

In this letter, we propose to use an optical system eye-mask parameterization technique to simplify the system performance evaluation process. This technique is based on the separation of distortion budget from noise budget. An optical eye distortion mask parameterization can be made at any reference interface of the system to define the distortion-related link performance contributions, independent of the particular noise characteristic.

\section{TheORETICAL ANALYSIS}

Fig. 1 is a typical eye diagram measured at the output of a dispersive fiber link with the noise averaged out. In Fig. 1, an eye-distortion mask is defined by a four-level feature $\left(P_{1} / P_{\text {av }}, P_{0} / P_{\text {av }}, A, B\right)$ over a timing window $W$ which

Manuscript received November 25, 1998; revised March 15, 1999.

R. Hui is with the Department of Electrical Engineering and Computer Science, University of Kansas, Lawrence, KS 66045 USA.

M. Vaziri, J. Zhou, and M. O'Sullivan are with Nortel Broadband Networks, Ottawa, ON K1Y 4H7, Canada.

Publisher Item Identifier S 1041-1135(99)05138-1.

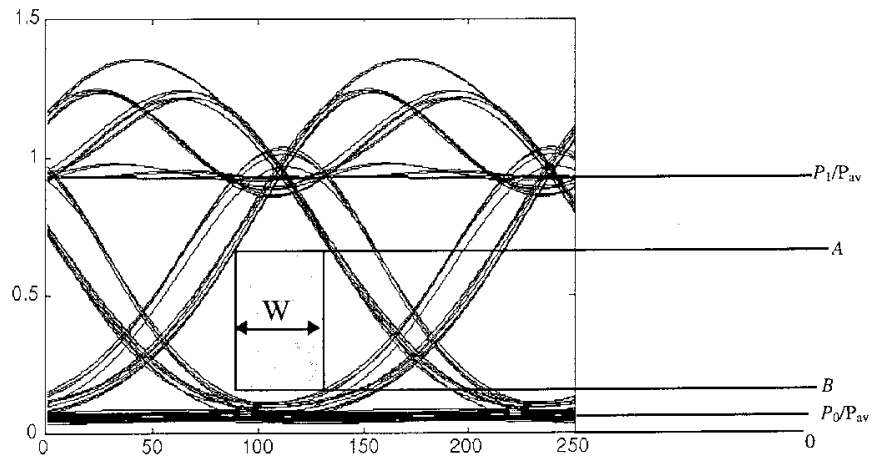

Fig. 1. Schematic of an optical eye-distortion-mask mapped onto a measured eye diagram.

represents the worst case phase uncertainty in the sampling instant. $W$ is comprised of the sum of all bounded uncertainties plus seven times the standard deviation of statistical uncertainty of the decision phase. The factor 7 was chosen to guarantee a bit error rate of $10^{-12} . P_{1}$ and $P_{0}$ are the power levels associated with signal long 1's and long 0 's in the pseudorandom bit pattern. The dimensionless parameters $A$ and $B$ are the lowest inner upper eye and the highest inner lower eye measured within the phase window $\mathrm{W}$, and they are independent of the noise. According to definitions in Fig. 1, the average signal optical power is $P_{\mathrm{av}}=\left(P_{1}+P_{0}\right) / 2$ giving that signal 1's and 0's have the same probability.

In practical systems, path distortion, signal-dependent noise and signal-independent noise are all mixed together at the receiver. The receiver $Q$ factor [3] $]^{1}$ can be written as

$$
Q=\frac{(A-B) 2 P_{\mathrm{av}}}{\sqrt{\sigma_{\mathrm{int}}^{2}+\eta A 2 P_{\mathrm{av}}}+\sqrt{\sigma_{\mathrm{int}}^{2}+\eta B 2 P_{\mathrm{av}}}}
$$

where, $\sigma_{\text {ind }}^{2}$ is the signal independent noise variance and $\eta$ is a system-dependent multiplication factor.

In the absence of distortion, $B=0$ and $A=1$, the system $Q$ is determined only by the noise contribution. In this case:

$$
Q=Q_{0}=\frac{2 P_{\mathrm{av}}}{\sqrt{\sigma_{\mathrm{int}}^{2}+\eta 2 P_{\mathrm{av}}}+\sqrt{\sigma_{\mathrm{int}}^{2}}} .
$$

By this definition of $Q_{0}$, the system $Q$ degradation caused by eye-distortion can be written in a general form as

$$
D(A, B, x)=\frac{Q}{Q_{0}}=\frac{A-B}{Y_{e}} .
$$

\footnotetext{
${ }^{1}$ See, e.g., [3, eq. (4.5.10)].
} 


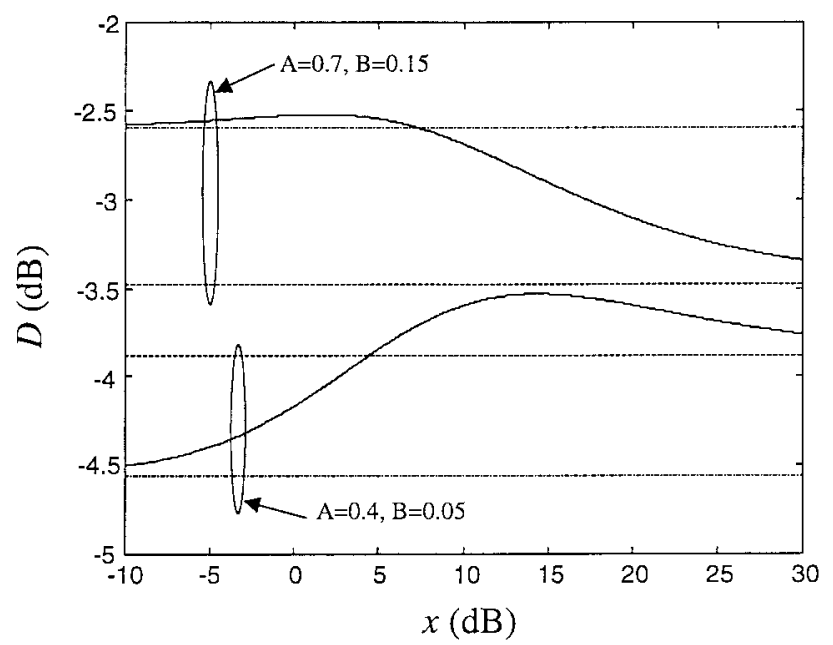

Fig. 2. $Q$-degradation parameter $D$ as a function of $10 \log (x)$ (solid line) for two different sets of $(A, B)$ values. Dashed lines: $10 \log (\sqrt{A}-\sqrt{B})$ and dashed-dotted lines: $10 \log (A-B)$.

In this expression, $Y_{e}$ is an important factor which shows the effect of interaction between distortion and noise,

$$
Y_{e}(A, B, x)=\frac{\sqrt{1+x A}+\sqrt{1+x B}}{1+\sqrt{1+x}}
$$

where $x=2 \eta P_{\mathrm{av}} / \sigma_{\int}^{2}$ is the ratio of signal-dependent noise to signal-independent noise.

In the case where signal independent noise dominates, $x=0$ and $Y_{e}=1$, so that $D=A-B$. On the other hand, if signal dependent noise dominates, $x=\infty$ and $Y_{e}=\sqrt{A}+\sqrt{B}$, therefore $D=\sqrt{A}-\sqrt{B}$. In general, with $x \in(0, \infty)$, the maximum value of $Y_{e}$, which corresponds to the worst-case distortion can be expressed as

$$
Y_{\max }= \begin{cases}\sqrt{A}+\sqrt{B}, & (\sqrt{A}+\sqrt{B}) \geq 1 \\ 1, & (\sqrt{A}+\sqrt{B})<1 .\end{cases}
$$

The two possible maximums $Y_{\max }=\sqrt{A}+\sqrt{B}$ and $Y_{\max }=1$ correspond to $x=\infty$ and $x=0$, respectively. Using (3), the worst-case distortion factor, defined as $D_{0}$, can be written as a function of $Y_{\max }$ :

$$
D_{0}=(A-B) / Y_{\max }
$$

Obviously, $D_{0}$ is a global worst-case distortion effect, which is independent of the nature of the noise.

In order to demonstrate the impact of noise characteristic on the system distortion penalty, $D$, defined by (3), is plotted in Fig. 2 as a function of $x$. In this plot, two sets of eye-closure parameters were used, corresponding to the conditions for the two solutions of (5). In one case, $A=0.7, B=0.15$ and $\sqrt{A}+\sqrt{B}>1$ so that the worst case distortion happens at $x=\infty$. In the other case, $A=0.4, B=0.05$, and $\sqrt{A}+\sqrt{B}<1$, the worst-case distortion happens at $x=0$. The dashed lines in Fig. 2 are $10 \log (\sqrt{A}-\sqrt{B})$ which represents the case where signal dependent noise dominates $(x=\infty)$, while the dashed-dotted lines are $10 \log (A-B)$, which represents the case of $x=0$. Shown as the solid lines

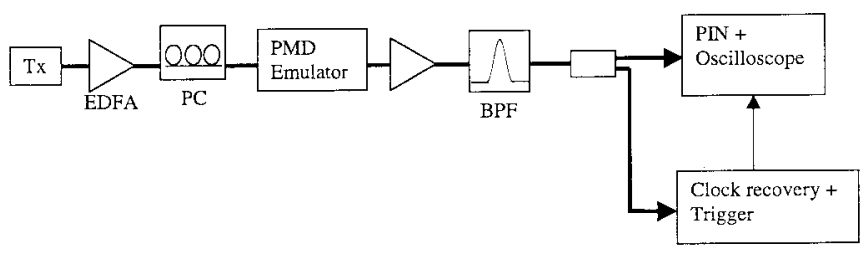

Fig. 3. Setup for PMD-induced distortion measurement. $T_{x}: 10-\mathrm{Gb} / \mathrm{s}$ optical transmitter. PC: Polarization control. BPF: Bandpass optical filter.

in Fig. 2, $D(x)$ versus $x$ characteristics are not monotonic, however, $D$ symptomatically approaches its worst-case $D_{0}$ with either $x=0$ or $x \rightarrow \infty$ depending on the value of $\sqrt{A}+\sqrt{B}$. Generally, (5) and (6) overestimate the distortion penalty because $x \in(0, \infty)$ was used to search for the worst case, but in real systems $x$ value can never be infinity.

Practically, the value of $x$ depends on the specific system configuration. In order to find a reasonable worst-case system distortion penalty, it is useful to know what is the highest $x$ value a practical system can have. Obviously, $x$ values are high in amplified optical systems where signal-spontaneous beat noise is the dominant noise. We have evaluated a large variety of optical systems with reasonable receiver parameters, $x$ values never exceeds 30 . Therefore, as an engineering design role, we choose $x=30$ as a conservative value to cover all realistic system configurations and receiver types [4]. So that, instead of using the global maximum $Y_{\max } x$, as defined by (5), it is more reasonable to use a realistic maximum of $Y_{e}$ value

$$
Y_{0}=\left.\max \left[Y_{e}(A, B, x)\right]\right|_{x \in(0,30)} .
$$

To evaluate the worst-case system distortion penalty

$$
D_{w c}=Q / Q_{0}=(A-B) / Y_{0}
$$

Evidently, $D_{w c}$ is a more realistic value for a practical system.

The success in finding the worst-case distortion factor $D_{w c}$ implies a possibility to separate distortion from noise in the system link budgeting. Equation (8) clearly demonstrates a simple linear relationship between system $Q$ and the worstcase distortion factor $D_{w c}$. Regardless of the fundamental difference in the origins of noise and distortion, isolation of these two gives a clear picture of system budget allocations.

\section{EXPERIMENTS}

In order to verify the linear relationship between $Q$ and $D_{w c}$, an experimental verification was carried out by adding a predetermined polarization-mode dispersion (PMD) to simulate the distortion in a fiber-optic system. In fact, PMD only introduces system eye-distortion but not noise, so, $Q_{0}$ does not change with the added PMD.

The experimental setup is shown in Fig. 3. A $10-\mathrm{Gb} / \mathrm{s}$ commercial transmitter was used to generate the optical signal. A polarization controller was placed between the amplified transmitter output and a PMD Emulator. ${ }^{2}$ The polarizationstate was adjusted such that $50 \%$ of the optical signal travels through each arm of the PMD emulator. Two EDFA's were used to increase optical signal power and a tunable bandpass

\footnotetext{
${ }^{2}$ JDS Fitel, PE3 PMD Emulator.
} 


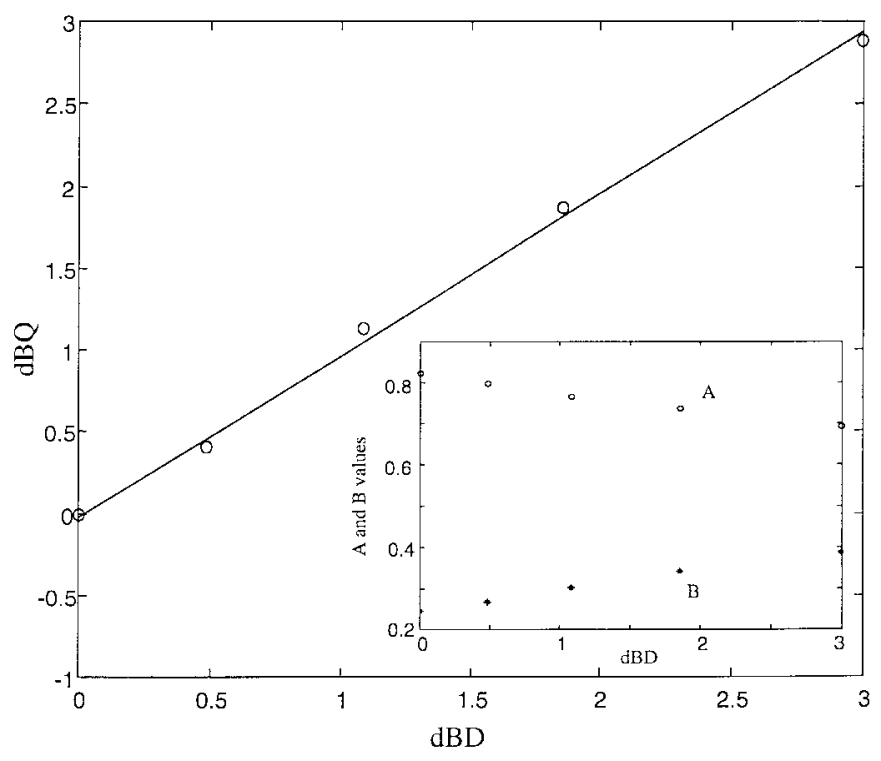

Fig. 4. Measured system penalty versus system distortion introduced by a variable PMD emulator. $Q$ and $D$ have been normalized by their values without PMD. Circles are the measured data and the solid line is the best fitting. The slope of the fitting line is $\Delta D_{w c}(\mathrm{~dB}) / \Delta Q(\mathrm{~dB})=0.99$. The jitter window $w$ used in the eye-mask measurement was 7 ps.

optical filter is used to remove wide-band ASE noise from EDFA's. At the receiver, the system $Q$ value was obtained by a BER-versus-decision-voltage (BERV) measurement [5]. The noise free signal waveform at the receiver was measured through the average of repeated $2^{7}-1$ bit patterns. Then the corresponding system distortion level $D_{w c}$ was evaluated through the measured signal waveforms using (7) and (8). The maximum $x$ value of 30 is always assumed as indicated in (7).
Fig. 4 shows the measured $10 \log (Q)$ versus $10 \log$. $\left[D_{w c}(A, B)\right]$ as defined in (8). The corresponding $A$ and $B$ values are also given in the inset of Fig. 4. A linear relationship between them was obvious. In fact, the slope of the best linear fit to this plot is 0.99 . Since optical power at the receiver was kept constant during the measurements $(-13$ $\mathrm{dBm}$ in this case) and thus $Q_{0}$ was constant, this verifies the theoretical prediction in (8). Note that the system was not calibrated to verify the $Q_{0}$ value as defined by (2), because this requires detailed information of all system components which was not accessible at the time of experiment.

\section{CONCLUSION}

We have proposed a novel method to separate optical systems' path-distortion from the random noise in the system budgeting. This greatly simplifies the system performance evaluation procedure and clarifies the origins of the system performance degradations. The method has been experimentally verified by introducing path distortions caused by PMD effect in a 10-Gb/s optical system.

\section{REFERENCES}

[1] I. P. Kaminow and T. L. Koch Eds., Optical Fiber Telecommunications IIIA. New York: Academic, 1997

[2] D. Marcuse, "Derivation of analytical expression for the bit-error probability in lightwave systems with optical amplifiers," J. Lightwave Technol., vol. 8, pp. 1816-1823, 1990.

[3] G. P. Agrawal, Fiber-Optic Communication Systems, 2nd ed. New York: Wiley, 1997.

[4] M. O'Sullivan, R. Hui, and J. Zhou, "Eye mask for measurement of distortion in optical transmission systems," U.S. patent 5774242 , June 1998.

[5] N. S. Bergano, K. W. Kerfoot, and C. R. Davidson, "Margin measurements in optical amplifier systems," IEEE Photonics Technol. Lett., vol. 5, pp. 304-306, 1993. 